\title{
POROCARCINOMA ECRINO: ESTUDIO CLIINICO- PATOLÓGICO DE 19 CASOS EN EL INSTITUTO NACIONAL DE ENFERMEDADES NEOPLÁSICAS, LIMA - PERÚ
}

\author{
Sandro Casavilca ${ }^{1, a}$, Anny Lama1,b, Melvy Guerrero ${ }^{1, a}$, Lydia R. Essary ${ }^{2,3, c}$, Raúl Mantilla $^{4, c}$, \\ Luis Cano ${ }^{1, e}$, Carlos Barrionuevo $0^{1, f}$
}

RESUMEN

Con el objetivo de describir las características clínico-patológicas de los casos de porocarcinoma ecrino (PE) registrados en el Instituto Nacional de Enfermedades Neoplásicas (INEN), se realizó un estudio trasversal que incluyó todos los casos entre 1998-2009. Para ello se realizó una revisión de fichas clínico-patológicas y una nueva lectura de las láminas de patología de los casos encontrados. Se hallaron 19 casos de PE; la mediana de edad fue de 64 años (rango: 37-98); con mayor frecuencia en el sexo masculino $(57,9 \%)$; la localización más frecuente fue en el pie $(21,1 \%)$, seguida de cabeza, tórax y muslo (15,8\%, en todos los casos). Se describe un caso de localización glútea que cursó con una conducta inusualmente invasiva y con compromiso rectal. Se concluye que el PE es una entidad poco frecuente en el Perú, su localización más común es en el pie, y afecta predominantemente al sexo masculino.

Palabras clave: Porocarcinoma ecrino; Neoplasias de las glándulas sudoríparas; Histopatología (fuente: DeCS BIREME).

\section{ECCRINE POROCARCINOMA: CLINICAL-PATHOLOGICAL STUDY OF 19 CASES IN THE INSTITUTO NACIONAL DE ENFERMEDADES NEOPLÁSICAS, LIMA - PERU}

\begin{abstract}
In order to describe the clinical-pathological characteristics of the cases of Eccrine Porocarcinoma (EP) found in the Instituto Nacional de Enfermedades Neoplásicas (INEN), a cross-sectional study was carried out, including all EP cases recorded between 1998 and 2009. A revision of the clinical-pathological records indentified and a new reading of the pathology slides of the cases found were performed. 19 EP cases were found, the median age of which was 64 years (range: 37-98). EP was primarily found on male patients $(57.9 \%)$ and was most commonly located on the foot $(21.1 \%)$, followed by the head, thorax and thigh (15.8\%, respectively). In one case, EP was located on the gluteal region, and showed an unusually invasive behavior and rectal involvement. We conclude that EP is a rather uncommon entity in Peru. Its most common location is on the foot and it affects mostly male patients for the most part.
\end{abstract}

Key words: Eccrine porocarcinoma; Sweat gland neoplasms; Histopathology (source: MeSH NLM).

\section{INTRODUCCIÓN}

Este cáncer fue descrito por primera vez por Pinkus y Mehregar en $1963^{(1,2)}$. Posteriormente, en 1969, Mishima y Morioka introdujeron el término porocarcinoma ecrino (PE). Desde entonces también ha sido llamado hidracantoma simple maligno, carcinoma de glándula sudorípara, siringoacantoma maligno o poroma displásico. Es el carcinoma de las glándulas sudoríparas más frecuente, pues representa entre el 0,005 a $0,01 \%$ de los tumores de la piel ${ }^{(3)}$. Se localiza con mayor más frecuencia en las extremidades inferiores ${ }^{(1,4)}$; aunque, se le puede encontrar también en la cabeza, el cuello, el tronco y el abdomen ${ }^{(1,5)}$.

En 2001, Robson et al. (1) describieron sus características histopatológicas señalando a su vez que ellas constituían factores pronósticos en la evolución de esta enfermedad, estas características enfatizaban el grado del tumor, basados en el pleomorfismo celular y la presencia de necrosis en los tumores de alto grado (Figura 1). Este cáncer puede hacer metástasis hacia ganglios linfáticos regionales y puede tener difusión pagetoide

\footnotetext{
Departamento de Patología, Instituto Nacional de Enfermedades Neoplásicas. Lima, Perú.

Cockerell \& Associates Dermpath Diagnostics. Dallas, EE. UU.

Department of Dermatology, University of Texas Southwestern Medical Center. Dallas, EE. UU.

Dirección de Educación, Instituto Nacional de Enfermedades Neoplásicas. Lima, Perú.

Médico patólogo; ${ }^{\mathrm{b}}$ médico radioterapeuta; ${ }^{\mathrm{c}}$ médico dermatopatólogo, doctor en Medicina; ${ }^{\mathrm{d}}$ estadístico; ${ }^{\mathrm{e}}$ médico cirujano; ${ }^{\mathrm{f}}$ médico patólogo, magister en oncología Molecular

Recibido: 16-01-13 Aprobado: 05-06-13
}

Citar como: Casavilca S, Lama A, Guerrero M, Essary LR, Mantilla R, Cano L, et al. Porocarcinoma ecrino: estudio clínico-patológico de 19 casos en el Instituto Nacional de Enfermedades Neoplásicas, Lima - Perú. Rev Peru Med Exp Salud Publica. 2013;30(3):437-40. 


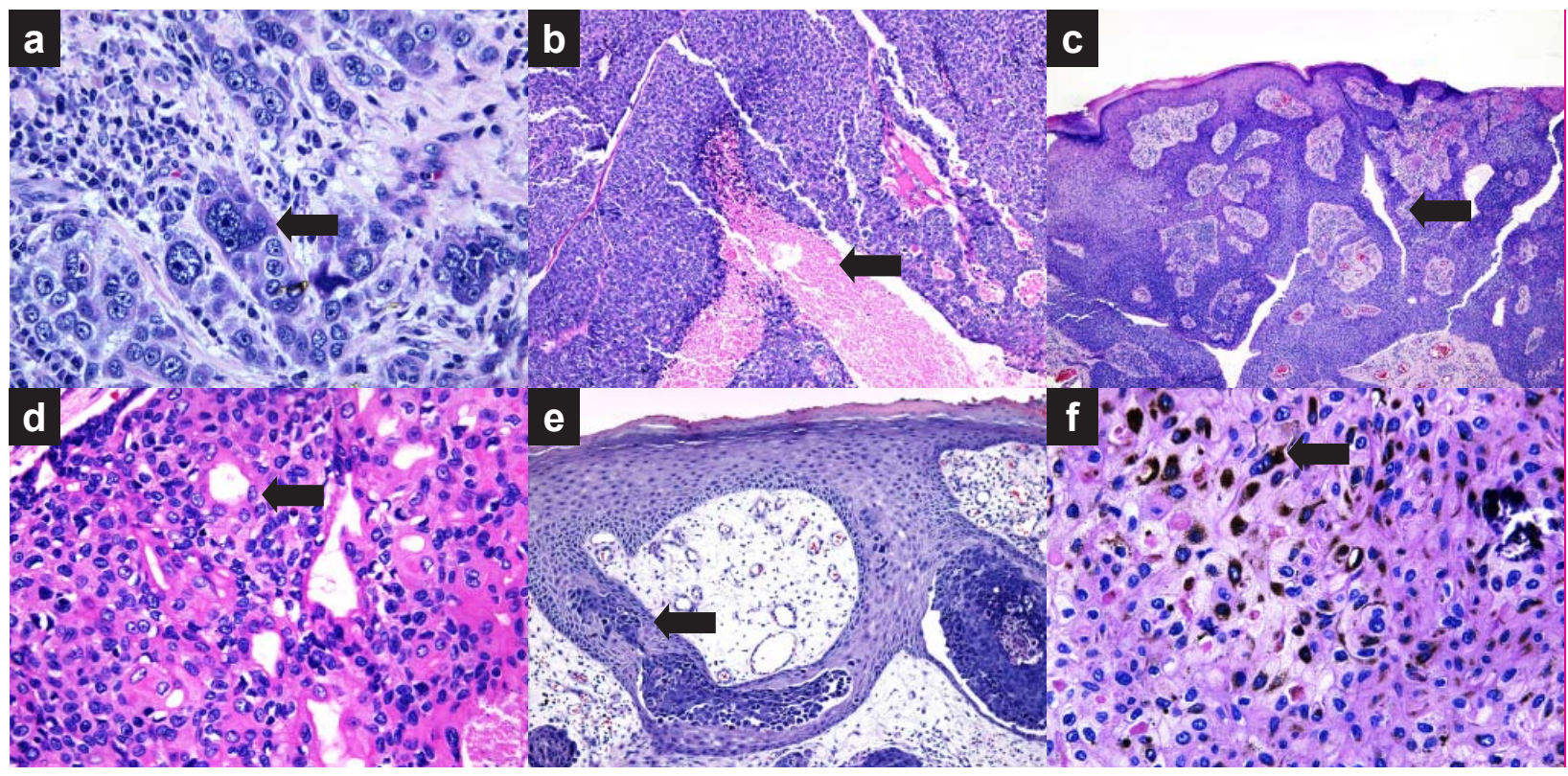

Figura 1. Criterios histológicos de anaplasia en porocarcinoma (señalados por las flechas)

a: pleomorfismo celular ( $\mathrm{HE}, 40 \mathrm{x})$, b: necrosis (HE, 10x), c: trabéculas epiteliales que se anastomosan (HE, 2,5x), d: diferenciación ductular bien definida (HE, 40x), e: pagetización intraepidermica (HE, 10x), f: pigmento melánico intraepitelial (HE, 40x)

intraepidérmica ${ }^{(5)}$. El PE es la contraparte maligna del poroma ecrino, del cual se diferencia por presentar pleomorfismo celular con hipercromatismo nuclear y actividad mitótica incrementada, en tanto que el poroma ecrino es un tumor simétrico y bien circunscrito, en el cual se puede encontrar atipia focal.

Debido a que se trata de un cáncer poco frecuente, existen escasos reportes respecto a él en la literatura biomédica, siendo la serie de Robson et al (1) de 2001, la más grande con 69 casos. En tanto que en Latinoamérica se han publicado reportes de algunos casos de presentación inusual (6-8). En el Perú, al igual que en el resto del mundo, el PE es una entidad infrecuente, y la información respecto a él es escasa. Es así que el objetivo de esta investigación fue describir las características clínico-patológicas de los casos de PE registrados en el Instituto Nacional de Enfermedades Neoplásicas (INEN) entre 1998 y 2009.

\section{EL ESTUDIO}

Se realizó un estudio transversal, para lo cual se revisó los archivos del INEN, se seleccionaron todos los casos de PE. Se realizó una revisión de fichas clínico-patológicas y una segunda revisión de las láminas de biopsia, este último proceso estuvo a cargo de dos patólogos expertos en patología tumoral de piel. Cuando se requirió confirmación diagnóstica, debido a que la tinción convencional con hematoxilina-eosina (H\&E) no fue suficiente, se tiñó adicionalmente las láminas con ácido peryódico de Schiff (PAS), anticuerpos para antígeno de membrana epitelial (EMA), y antígeno carcinoembrionario (CEA). Se evaluaron las características histopatológicas procurando identificar los factores pronósticos, descritos en el reporte de Robson (1). El plan de análisis estadístico incluyó el cálculo de frecuencias tanto absolutas, como relativas, y las medidas de resumen para las variables en estudio. Para este análisis se empleó el paquete estadístico R. Se contó con la aprobación del Comité de Investigación del Instituto Nacional de Enfermedades Neoplásicas.

\section{HALLAZGOS}

Se identificaron diecinueve casos diagnosticados como PE en los archivos del INEN, entre los años 1998 y 2009. En 16 de los casos se tuvieron láminas de secciones histológicas teñidas con H\&E. En cuatro de ellas se requirió la tinción adicional con PAS, EMA y CEA. Dos de los casos correspondieron a consultas de otras instituciones por lo que no se contó con secciones histológicas disponibles para su revisión. Uno de los casos fue encontrado como parte del ingreso del paciente a la institución, sin reporte de grado y sin láminas histológicas ni bloque de parafina para revisión, por haberse devuelto el material a la institución de donde vino referido. Ninguno de estos pacientes fue excluido del análisis. 
Tabla 1. Características clínico-patológicas de pacientes con diagnóstico de porocarcinoma en el INEN, 19992008.

\begin{tabular}{|c|c|}
\hline Características & N. ${ }^{\circ}(\%)$ \\
\hline Edad (años)* & $64,0(37-98)$ \\
\hline \multicolumn{2}{|l|}{ Sexo } \\
\hline Hombre & $11(57,9)$ \\
\hline Mujer & $8(42,1)$ \\
\hline \multicolumn{2}{|l|}{ Localización } \\
\hline $\mathrm{Pie}$ & $4(21,1)$ \\
\hline Tórax & $3(15,8)$ \\
\hline Muslo & $3(15,8)$ \\
\hline Cabeza & $3(15,8)$ \\
\hline Pierna & $2(10,5)$ \\
\hline Glúteo & $2(10,5)$ \\
\hline Axila & $1(5,3)$ \\
\hline Brazo & $1(5,3)$ \\
\hline Evolución (años) * & $3,0(0,06-34,0)$ \\
\hline Tamaño $(\mathrm{cm})$ * & $3,0(1-10)$ \\
\hline \multicolumn{2}{|l|}{ Asimetría } \\
\hline Sí & $13(68,4)$ \\
\hline No & $3(15,8)$ \\
\hline No precisable & $3(15,8)$ \\
\hline \multicolumn{2}{|l|}{ Bordes } \\
\hline Definidos & $10(52,6)$ \\
\hline Infiltrativos & $4(21,1)$ \\
\hline No precisable & $5(26,3)$ \\
\hline \multicolumn{2}{|l|}{ Grado } \\
\hline Bajo & $6(35,3)$ \\
\hline Alto & $10(58,8)$ \\
\hline No precisable & $1(5,9)$ \\
\hline Recuento mitótico (x10 CAP)* & $16,0(5-66)$ \\
\hline \multicolumn{2}{|l|}{ Márgenes laterales } \\
\hline Libres & $8(47,1)$ \\
\hline Comprometidos & $3(17,6)$ \\
\hline Biopsia & $6(35,3)$ \\
\hline \multicolumn{2}{|l|}{ Márgenes profundos } \\
\hline Libres & $8(47,1)$ \\
\hline Comprometidos & $2(11,8)$ \\
\hline Biopsia & $6(35,3)$ \\
\hline No evaluables & $1(5,9)$ \\
\hline Infiltración (mm)* & $8,75(2-25)$ \\
\hline \multicolumn{2}{|l|}{ Patrón de infiltración } \\
\hline Expansivo & $10(58,8)$ \\
\hline Infiltrativo & $5(29,4)$ \\
\hline No precisable & $2(11,8)$ \\
\hline
\end{tabular}

El $57,9 \%(11 / 19)$ de los pacientes fueron de sexo masculino; la mediana de edad fue de 64 años (rango 37-98); el 47,4\% (9/19) de los PE tuvo como localización las extremidades inferiores (pies, piernas y muslos); y la media de tamaño fue de $3,4 \mathrm{~cm}$. Los tumores polipoides fueron los más frecuentes $(47,4 \%$ [9/09]), y en el 68,4\% (13/19) de los casos se observó ulceraciones. Uno de los casos de localización glútea cursó con un comportamiento localmente agresivo y compromiso rectal, otras características clínicas se detallan en la Tabla 1. Con relación a la caracterización histopatológica, el 58,8\% (10/19) de los casos fueron clasificados de alto grado, basados en los criterios de Robson (1), y en el 36,8\% (7/19) de los casos se encontró pigmento melanocítico intraepitelial. La mediana de recuento mitótico fue de 16 recuentos por 10 campos de alto poder (CAP) (rango: 5-66), en los PE de alto grado el recuento mitótico tuvo una mediana de 36 recuentos por 10 CAP (rango: 9-66), para los tumores de bajo grado la mediana del recuento mitótico fue de 10 recuentos por 10 CAP (rango: 5-16). La mediana de la profundidad de la infiltración fue de $8,8 \mathrm{~mm}$ (rango 2-25). En el $21 \%$ (4/19) de los casos se encontró metástasis a ganglios linfáticos, todos ellos en el grupo de PE de alto grado. La mediana de tiempo de enfermedad fue de 1,5 años (rango $0,1-15$ ); en tanto que para los $\mathrm{PE}$ de bajo grado la mediana del tiempo de enfermedad fue de 26,5 años (rango 2,0-34,0)

El manejo fue quirúrgico en todos los casos, con resección local en el 73,7\% (14/19) de ellos; en tanto que en el $26,3 \%$ (5/19) solo se obtuvo una biopsia inicial. La media del seguimiento fue 10 meses. Respecto a la evolución de los pacientes, el 15,8\% (3/19) de ellos no retornó después del diagnóstico; el 10,5\% (2/19) falleció a causa de esta enfermedad; el 5,2\% (1/19) presento recurrencia a los 29 meses, y en otro $5,2 \%$ de los casos se encontró persistencia de la lesión.

\section{DISCUSIÓN}

El porocarcinoma es un tumor de las glándulas sudoríparas que se cree se origina del acrosiringio y se clasifica en dos entidades, aquellos de localización intraepidérmica, también conocido como porocarcinoma in situ o hidroacantoma simple maligno, y la variante invasiva o porocarcinoma. Los archivos de patología en el INEN contienen diecinueve casos de porocarcinoma ecrino diagnosticados en esta institución desde 1998 al 2009. Todos ellos eran pacientes peruanos, la relación hombre-mujer encontrada fue de 1,4 a 1, a diferencia de otros estudios que reportan una discreta predominancia femenina (1). Las características patológicas fueron correlacionadas con el comportamiento clínico y las metástasis a ganglios linfáticos. La metástasis ganglionar estuvo presente en el $21 \%$ de los casos, todos ellos tumores de alto grado. Del mismo modo, el alto grado histológico se correlacionó con un índice mitótico alto en comparación con el grupo de bajo grado.

Durante el estudio se encontró un caso de localización glútea y conducta invasiva, esta ubicación y comportamiento son poco usuales en el PE y, eventualmente, no se han encontrado reportes similares. La lesión correspondió a una mujer de 37 años, con un tiempo de enfermedad de 2 años, quien al examen clínico presentaba un tumor en el glúteo derecho, en donde se podía distinguir una cicatriz quirúrgica cercana a la 
región lumbosacra, alrededor de la cual se encontraba una tumoración de $10 \mathrm{~cm}$, se practicó a la paciente una resección subtotal. Al realizar la sección del musculo glúteo, se evidenció que la tumoración era mixta con secreción líquida y gran componente sólido, la cual se extendía hasta la zona presacra, no se logró identificar el límite debido a la gran cantidad de vasos y por el sangrado que presentaba, por ello solo se extrajo un $90 \%$ de la lesión, quedando enfermedad macroscópica. El estudio patológico informó que la lesión correspondía a un carcinoma poco diferenciado consistente con porocarcinoma. El tumor medía $8,5 \mathrm{~cm}$ y comprometía en profundidad el tejido celular subcutáneo con necrosis extensa. Tras 25 sesiones de radioterapia, la paciente cursó con sangrado rectal, anemia, dolor intenso a nivel de zona coccígea, y crecimiento tumoral a ese nivel, tras lo cual la paciente descontinúa su tratamiento en el INEN.

Otro aspecto a destacar, es que tres de los casos encontrados fueron previamente diagnosticados en su institución de origen como carcinoma basocelular, y dos casos como carcinoma epidermoide poco diferenciado; por cuanto, es posible afirmar que existiría una alta tasa de subdiagnóstico de casos de PE, hecho que cobra mayor importancia si se toma en cuenta que se trata de un cáncer que es potencialmente curable quirúrgicamente, aun cuando en la literatura se señala que la metástasis estaría presente en el 19 al 25\% de los casos (1,4); siendo, por tanto de suma importancia el diagnóstico preciso y el establecimiento de criterios pronósticos como el grado histológico.
Algunas limitaciones del estudio deben ser reconocidas pues al tratarse de una enfermedad de baja prevalencia el número de casos es limitado, por cuanto no se ha podido realizar una análisis estadístico más profundo. Otra limitación es el hecho que los datos clínicos se han obtenido de manera indirecta, consideramos, sin embargo, que al ser el INEN el establecimiento de referencia nacional, el número de casos encontrados da una buena idea de las características del PE en la población peruana.

En conclusión, el PE es una neoplasia poco frecuente en el Perú, cuya localización más frecuente es en miembros inferiores, particularmente en el pie, y que afecta con más frecuencia a los varones. Existe la posibilidad, además, de un subdiagnóstico de este cáncer, por lo que es necesario tomar en cuenta al $\mathrm{PE}$ dentro de las posibilidades diagnósticas de las neoplásicas de la piel, para poder hacer un diagnóstico oportuno.

Contribuciones de autoría: SC participó de la concepción, diseño del estudio, revisión de las láminas de patología y redacción del primer borrador del artículo. AL y MG participaron de la revisión de historias clínicas y llenado de las fichas clínicas y patológicas, LRE participó de la revisión crítica del artículo, RM participó de la asesoría estadística, CB participó de la revisión de láminas de patología. Todos los autores aprobaron la versión corregida del artículo.

Conflictos de interés: los autores declaran no tener conflictos de interés.

Fuentes de financiamiento: autofinanciado.

\section{REFERENCIAS BIBLIOGRÁFICAS}

1. Robson A, Greene J, Ansari N, Kim B, Seed PT, McKee PH, et al. Eccrine porocarcinoma (malignant eccrine poroma) a clinicopathologic study of 69 cases. Am J Surg Pathol. 2001;25(6):710-20.

2. Yu L, Olsen S, Lowe L, Michael C, Jing X. Fine-needle aspiration cytology of metastatic eccrine porocarcinoma. Diagn Cytopathol. 2009;37(10):7558. doi: $10.1002 / \mathrm{dc} .21099$.

3. Chang NC, Tsai KB. Eccrine porocarcinoma of the auricle: a case report. Kaohsiung J Med Sci. 2009;25(7):401-4. doi: 10.1016/ S1607-551X(09)70534-0.

4. Shiohara J, Koga H, Uhara H, Takata M, Saida T. Eccrine porocarcinoma: clinical and pathological studies of 12 cases. J Dermatol. 2007;34(8):516-22.

5. González-López MA, Vázquez-López F, Soler T, Gómez-Diéz S, Garcia YH Manjón JA, et al. Metastatic eccrine porocarcinoma: a 5.6-year followup study of a patient treated with a combined therapeutic protocol. Dermatol Surg. 2003;29(12):1227-32.

6. Sanz ME, Herrera E, Arias Stella Castillo J, Arias Stella J. Porocarcinoma ecrino en una paciente embarazada. Folia dermatol Peru. 2007;18(2):8992.

7. Lannicelli E, Galluzzo A, Salvi P, Ziparo V, David V. A large porocarcinoma of perineal region: MR findings and review of the literature. Abdom
Imaging. 2008;33(6):744-7. doi: 10.1007/s00261-007-9356-4.

8. Asghar AH, Mahmood H, Faheem M, Rizvi S, Khan KA, Irfan J. Porocarcinoma: a rare sweat gland malignancy. J Coll Physicians Surg Pak. 2009;19(6):389-90. doi: 06.2009/ JCPSP.389390.

Correspondencia: Sandro Casavilca Zambrano Dirección: Instituto Nacional de Enfermedades Neoplásicas, Departamento de Patología, Oficina 155

Av. Angamos Este 2520, Lima 34, Perú. Teléfono: (511) 2016500 Anexo 1410.

Correo electrónico:sandrocasavilca@hotmail.com 